\title{
SOLUTION OF FREE HARMONIC VIBRATION EQUATION OF SIMPLY SUPPORTED KIRCHHOFF PLATE BY GALERKIN-VLASOV METHOD
}

\author{
B. O. Mama ${ }^{1}$, H. N. Onah ${ }^{2}$, C. C. Ike ${ }^{3,{ }^{*}}$ and N. N. Osadebe ${ }^{4}$ \\ 1, 2,4DEPARTMENT OF Civil ENGinEERING, UNIVERSity of NigeRIA, NSUKKA. ENUGU STATE. NIGERIA

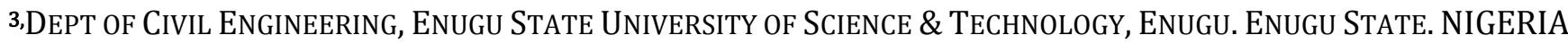 \\ E-mail addresses:1 benjamin.mama@unn.edu.ng, ${ }^{2}$ hyginus.onah@unn.edu.ng,3ikece2007@yahoo.com, \\ 4 mkemamaka@yahoo.com
}

\begin{abstract}
This work studies the dynamic characteristics of simply supported rectangular thin plates undergoing natural transverse vibrations in harmonic motion. The governing partial differential equation for the free transverse vibration of the plate was solved by the Galerkin-Vlasov variational technique. The assumption of free harmonic motions reduced the governing equation to an algebraic eigen value eigenvector problem, which was solved in the space domain to obtain the eigen frequencies and modal shape functions of the vibrating Kirchhoff plate. The eigen frequencies and modal shape functions obtained were found to be identical with the results obtained by the classical methods of Navier and Levy for the same problem.
\end{abstract}

Keywords: Kirchhoff plate, Galerkin-Vlasov method, harmonic vibration, natural vibrations, eigen frequencies.

\section{INTRODUCTION}

The vibratory characteristics of rectangular thin plates with various edge support conditions are of considerable importance in all the fields of structural, mechanical, aerospace, aeronautical and geotechnical engineering [1 - 5]. The free vibration analysis of rectangular plates is significant for controlling the resonance, thus ensuring the safety of plates. The vibration of plates is thus important in the study of practical structural systems such as bridge decks, ship decks, airplanes, machine parts, highway pavements, airport runways and floor slabs.

The theory of elastic plates is an approximation of the three dimensional elasticity theory to two dimensions, which permits a description of the deformation field in the plate in terms of the deformation of the middle surface of the plate $[1,6]$. The equations of motion of plates have been derived using equilibrium approach and variational approach and based on small deflection, thin plate assumption, and thick plate assumptions by [7 - 12]. Mindlin and Reissner plate theories consider the effects of rotary inertia and shear deformation, which are disregarded in the Kirchhoff plate theory $[1-3]$. The dynamic plate problem has been studied using the classical technique of Navier and Levy, based on assuming displacement shape function in the form of double trigonometric (Fourier) series, and single trigonometric (Fourier) series, respectively[14, 5]. Solutions obtained on applying the boundary conditions yield the natural frequencies and mode shapes of the vibrating plate, which values are used in the computation of dynamic stresses caused by external excitations. Classical techniques of Navier and Levy are not applicable in some plate problems, necessitating approximate solutions of the eigen value problem. Some of the approximate methods used in dynamic plate problems are Raleigh Method, Raleigh - Ritz Method, Finite Element Method, Finite Difference Method and Variational Methods of Galerkin [15] and modifications by Kantorovich and Bubnov [5, 6, 14]. Another method is the integral transform methods [16].

\section{ADVANTAGES OF THE GALERKIN-VLASOV METHOD}

The Galerkin-Vlasov method is used in this work because of the advantages namely:

(i) the method is generally rapidly converging and is and efficient computational method

(ii) the solution of complex plate problems is simplified to the evaluation of certain definite integrals which can be done numerically; in cases where closed form analytical integration is impossible or difficult.

(iii) it is closely related to the Rayleigh-Ritz and finite element methods 
(iv) due to the relative ease in selecting shape functions for most boundary conditions, Galerkin-Vlasov method is considered more universal than other variational methods

(v) further simplification in the numerical computation of the Vlasov's method is achieved due to the quasiorthogonality of the eigen functions of the vibrating Euler-Bernoulli beam used as the shape functions in the $x$-direction.

(vi) Galerkin Vlasov's method greatly reduces the amount of computational and numerical work needed by the use of eigen functions of vibrating beams as the basis functions due to the orthogonal properties and behaviour of the eigen functions.

(vii) the choice of an appropriate basis function simply reduces to a matter of choosing beam functions with identical boundary conditions as those of the plate.

(viii) the solution becomes increasingly tedious and tasking as the number of unknown parameters is increased. However, the results indicate that in general, only a few number of unknown parameters is required to produce acceptable solutions.

(ix) Galerkin-Vlasov method is useful in the manual solutions of plate problems.

\section{MATHEMATICAL MODEL}

The rectangular Kirchhoff plate shown in Fig 1 is considered. The governing partial differential equation for vibrating Kirchhoff plates is the fourth order elliptic in homogeneous equation.

$$
D \nabla^{4} w(x, y, t)+\rho h w_{t t}=P_{Z}(x, y, t)
$$

where $w(x, y, t)$ is the dynamic displacement, $w_{t t}$ is the second time derivative of the dynamic displacement, $\rho$ is the mass density, $h$ is the plate thickness, $D$ is plate flexural rigidity, $p_{z}(x, y, t)$ is the externally applied vibratory load, $D=E h^{3} / 12\left(1-\mu^{2}\right), \quad E$ is Young's modulus of elasticity and $\mu$ is Poisson's ratio.

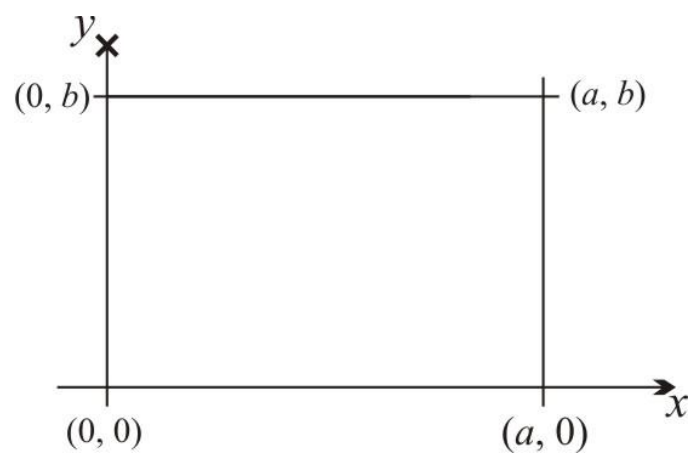

Fig 1: Rectangular Kirchhoff plate

The boundary conditions for simply supported plate edges $x=0, a, y=0, b$, are

$$
w=0 \text {, on } x=0, x=a
$$

$$
\begin{aligned}
& w=0 \text { on } \mathrm{x}=0, \quad \mathrm{x}=\mathrm{a} \\
& w=0 \text { on } \mathrm{y}=0, \quad \mathrm{y}=\mathrm{b} \\
& w_{x x}=0 \text { on } \mathrm{x} 0, \quad \mathrm{x}=\mathrm{a} \\
& w_{y y}=0 \text { on } \mathrm{y}=0, \mathrm{y}=\mathrm{b} \\
& \text { where } \quad w_{x x}=\frac{\partial^{2} w}{\partial x^{2}}, w_{y y}=\frac{\partial^{2} w}{\partial y^{2}}
\end{aligned}
$$

Let the initial displacement and initial velocity of the plate be given by

$$
\begin{aligned}
& w(x, y, 0)=w_{0}(x, y) \\
& \dot{w}(x, y, 0)=\dot{w}_{0}(x, y)
\end{aligned}
$$

where the dot over $w(x, y, 0)$ represent time derivative. The boundary value problem presented by the governing equation for free vibration of Kirchhoff plates has been solved using Navier's method for simply supported rectangular plates by Timoshenko, who obtained the natural frequencies as:

$$
\omega_{m n}=\pi^{2}\left(\frac{m^{2}}{a^{2}}+\frac{n^{2}}{b^{2}}\right) \sqrt{\frac{D}{\rho h}}
$$

where $m=1,2,3,4, .$.

$n=1,2,3,4, \ldots$

and the modal shape functions as

$$
\varphi_{m n}=\sin \frac{m \pi x}{a} \sin \frac{n \pi y}{b}
$$

Timoshenko and Woinowsky-Krieger also presented the same results for simply supported thin plates using Levy method.

\section{APPLICATION OF THE GALERKIN-VLASOV METHOD}

For harmonic excitation, we assume a harmonic response, let:

$$
w(x, y, t)=W(x, y) \varphi(t)
$$

In (6), $W(x, y)$ is the displacement function of the vibrating plate $\varphi(t)$ is function describing the time dependency of the displacement of the vibrating plate. $\varphi(t)$ is assumed to vary harmonically with time. Hence, we let $\varphi(t)=\exp i \omega t$, then

$$
w(x, y, t)=W(x, y) e^{i \omega t}
$$

and the BVP becomes

$$
\begin{gathered}
D \nabla^{4} W e^{i \omega t}-\rho h \omega^{2} W e^{i \omega t}=P_{z}(x, y, t) \\
\left(\nabla^{4} W-\frac{\rho h \omega^{2} W}{D}\right) e^{i \omega t}=\frac{p(x, y, t)}{D} \\
\left(\nabla^{4} W-\lambda^{4} W\right) e^{i \omega t}=\frac{p(x, y, t)}{D} \\
\text { where } \lambda^{4}=\frac{\rho h \omega^{2}}{D}
\end{gathered}
$$

For free vibration,

$$
\begin{array}{ll} 
& \left(\nabla^{4} W-\lambda^{4} W\right) e^{i \omega t}=0 \\
& \left(\nabla^{4} W-\lambda^{4} W\right)=0 \\
& \left(\nabla^{4}-\lambda^{4}\right) W(x, y)=0 \\
\text { Let } \quad & W(x, y)=\sum_{m}^{\infty} \sum_{n}^{\infty} a_{m n} \varphi_{m n}(x, y)
\end{array}
$$


$a_{m n}$ is the displacement amplitudes, $\varphi_{m n}$ is the displacement shape (basis) functions.

For simply supported plates.

$$
\begin{gathered}
\varphi_{m n}=\sin \alpha_{m} x \sin \beta_{\mathrm{n}} \mathrm{y} \\
\text { where } \alpha_{m}=m \pi / a, \beta_{n}=n \pi / b
\end{gathered}
$$

The Galerkin-Vlasov variational integral becomes:

$$
\begin{aligned}
\int_{0}^{b} \int_{0}^{a} \nabla^{4}\left(\sum_{m=1}^{\infty} \sum_{n=1}^{\infty} a_{m n} \varphi_{m n}\right. \\
\left.-\lambda^{4} \sum_{m=1}^{\infty} \sum_{n=1}^{\infty} a_{m n} \varphi_{m n}\right) \varphi_{r s} d x d y \\
=0
\end{aligned}
$$

$\sum_{m}^{\infty} \sum_{n}^{\infty} \iint \varphi_{r s}\left(\nabla^{4} a_{m n} \varphi_{m n}\right) d x d y$

$$
-\lambda^{4} \sum_{m}^{\infty} \sum_{n}^{\infty} \varphi_{m n} \int_{0}^{b} \int_{0}^{a} \varphi_{m n} \varphi_{r s} d x d y
$$$$
=0
$$

$$
\begin{aligned}
\sum_{m}^{\infty} \sum_{n}^{\infty} a_{m n} \int_{0}^{b} \int_{0}^{a} \varphi_{r s}\left(\nabla^{4} \varphi_{m n}\right) d x d y & \\
& \quad-\lambda^{4} \sum_{m}^{\infty} \sum_{n}^{\infty} \alpha_{m n} \int_{0}^{b} \int_{0}^{a} \varphi_{m n} \varphi_{r s} d x d y
\end{aligned}
$$$$
=0
$$

In general,

$$
\begin{aligned}
& \left\{(K)-\lambda^{4}(M)\right\}\{x\}=0 \\
& (K)=K_{r s_{m n}}=\int_{0}^{b} \int_{0}^{a} \varphi_{r s} \nabla^{4} \varphi_{m n} d x d y \\
& (M)=M_{r s_{m n}}=\int_{0}^{b} \int_{0}^{a} \varphi_{r s} \varphi_{m n} d x d y
\end{aligned}
$$

$K_{r s_{m n}}=\int_{0}^{b} \int_{0}^{a} \sin \alpha_{\mathrm{r}} x \sin \beta_{s} y\left(\nabla^{4} \sin \alpha_{\mathrm{m}} x \sin \beta_{m n}\right) d x d y$

$K_{\text {rsmn }}$

$=\int_{0}^{b} \int_{0}^{a}\left(\alpha_{m}^{2}\right.$

$\left.+\beta_{n}^{2}\right)^{2} \sin \alpha_{\mathrm{r}} x \sin \beta_{s} y \sin \alpha_{m} x \sin \beta_{n} y d x d y$

$K_{\text {rsmn }}$

$=\left(\alpha_{m}^{2}+\beta_{n}^{2}\right)^{2} \int_{0}^{b} \int_{0}^{a} \sin \alpha_{\mathrm{r}} x \sin \alpha_{m} x \sin \beta_{s} y \sin \beta_{n} y d x d y$

$K_{\text {rsmn }}=\left(\alpha_{m}^{2}+\beta_{n}^{2}\right)^{2} \int_{0}^{b} \int_{0}^{a} \sin ^{2} \alpha_{\mathrm{m}} \sin ^{2} \quad \beta_{n} y d x d y$

$K_{r s m n}=\left(\alpha_{m}^{2}+\beta_{n}^{2}\right) \frac{a b}{4} \quad r=m s=n$

$$
K_{r s m n}=0(r \neq m, s \neq n)
$$

$M_{r s m n}=\int_{0}^{b} \int_{0}^{a} \sin a x \sin \beta_{n} y \sin \alpha_{m} x \sin \beta_{n} y d x d y$

$$
\begin{array}{cl}
M_{r s m n}=\int_{0}^{b} \int_{0}^{a} \sin ^{2} & \alpha_{m} x \sin ^{2} \beta_{n} y d x d y \\
M_{r s m n}=\frac{a b}{4} & r=m, \quad s=n \\
M_{r s m n}=0 & r \neq m, s \neq n
\end{array}
$$

Thus

$$
\begin{gathered}
\left(\frac{a b}{4}\left(\alpha_{m}^{2}+\beta_{n}^{2}\right)^{2}-\lambda^{4} \frac{a b}{4}\right) a_{m n}=0 \\
\left(\alpha_{m}^{2}+\beta_{n}^{2}\right)^{2}-\lambda^{4}=0 \\
\lambda^{4}=\left(\alpha_{m}^{2}+\beta_{n}^{2}\right)^{2} \\
\lambda^{4}=\left(\left(\frac{m \pi}{a}\right)^{2}+\left(\frac{n \pi}{b}\right)^{2}\right)^{2}=\pi^{4}\left(\left(\frac{m}{a}\right)^{2}+\left(\frac{n}{b}\right)^{2}\right)^{2} \\
\omega_{m n}^{2} \frac{\rho h}{D}=\pi^{4}\left(\frac{m^{2}}{a^{2}}+\frac{n^{2}}{b^{2}}\right)^{2} \\
\omega_{m n}^{2}=\frac{D \pi^{4}}{\rho h}\left(\frac{m^{2}}{a^{2}}+\frac{n^{2}}{b^{2}}\right)^{2} \\
\omega_{m n}=\sqrt{\frac{D}{\rho h}} \pi^{2}\left(\frac{m^{2}}{a^{2}}+\frac{n^{2}}{b^{2}}\right) \\
\omega_{m n}=\left(\alpha_{m n}^{2}+\beta_{m n}^{2}\right) \sqrt{\frac{D}{\rho h}}
\end{gathered}
$$

$\omega_{m n}$ is the natural frequency of harmonic vibration of Kirchhoff plate. The displacement for free vibrations are:

$$
\begin{aligned}
& w(x, y, t)=\sum_{m}^{\infty} \sum_{n}^{\infty} a_{m n} \sin \frac{m \pi x}{a} \sin \frac{n \pi y}{b}\left(\mathrm{c}_{1} \sin \omega \mathrm{t}\right. \\
& \left.+c_{2} \cos \omega t\right) \\
& w(x, y, 0)=w_{0}=\sum_{m}^{\infty} \sum_{n}^{\infty} a_{m n} \sin \frac{m \pi x}{a} \sin \frac{n \pi y}{b}\left(\mathrm{c}_{1} \sin 0\right. \\
& \left.+\mathrm{c}_{2}\right)=\mathrm{w}_{0}(\mathrm{x}, \mathrm{y}) \\
& \therefore w_{0}(x, y,)=\sum_{m}^{\infty} \sum_{n}^{\infty} a_{m n} c_{2} \sin \frac{m \pi x}{a} \sin \frac{n \pi y}{b}
\end{aligned}
$$

$\dot{w}(x, y, 0)$

$=\sum_{m}^{\infty} \sum_{n}^{\infty} a_{m n} \sin \frac{m \pi x}{a} \sin \frac{n \pi y}{b} \mathrm{c}_{1} \omega=\dot{\mathrm{w}}_{0}(x, y)$

$=\sum_{m}^{\infty} \sum_{n}^{\infty} a_{m n} \mathrm{c}_{1} \omega \sin \frac{m \pi x}{a} \sin \frac{n \pi y}{b}$

$a_{m n} \mathrm{c}_{1} \omega=\frac{4}{a b} \int_{0}^{b} \int_{0}^{a} \dot{w}_{0}(x, y,) \sin \frac{m \pi x}{a} \sin \frac{n \pi y}{b} d x d y$ 


$$
\begin{aligned}
a_{m n} \mathrm{c}_{1}=\frac{4}{a b \omega} \int_{0}^{b} \int_{0}^{a} \dot{\omega}_{0}(x, y,) \sin \frac{m \pi x}{a} \sin \frac{n \pi y}{b} d x d y & (48) \\
w(x, y, t)= & \sum_{m}^{\infty} \sum_{n}^{\infty}\left(\frac{4}{a b \omega} \int_{0}^{b} \int_{0}^{a} \dot{w}_{0}(x, y,) \sin a_{m} x \sin \beta_{n} y d x d y\right) \sin \omega t \sin \alpha_{m} x \sin \beta_{n} y \\
& +\sum_{m}^{\infty} \sum_{n}^{\infty}\left(\frac{4}{a b} \int_{0}^{b} \int_{0}^{a} \dot{w}_{0}(x, y,) \sin a_{m} x \sin \beta_{n} y d x d y\right) \cos \omega t \sin a_{m} x \sin \beta_{n} y
\end{aligned}
$$

\begin{tabular}{|c|c|c|c|}
\hline$m$ & $n$ & $\lambda_{m n}^{2}$ & mode shape \\
\hline 1 & 1 & 389.64 & $\begin{array}{cc}\sin \frac{\pi x}{a} \sin \frac{\pi y}{b} \\
\pi x & 2 \pi y\end{array}$ \\
\hline 1 & 2 & 2435.23 & $\sin \frac{\bar{a}}{\sin } \frac{b}{b}$ \\
\hline 2 & 1 & 2435.23 & $\sin \frac{2 \pi x}{a} \sin \frac{\pi y}{b}$ \\
\hline 2 & 2 & 6234.18 & $\sin \frac{2 \pi x}{a} \sin \frac{2 \pi y}{b}$ \\
\hline 1 & 3 & 9740.91 & $\sin \frac{\pi x}{a} \sin \frac{3 \pi y}{b}$ \\
\hline 3 & 1 & 9740.91 & $\sin \frac{3 \pi x}{a} \sin \frac{\pi y}{b}$ \\
\hline 2 & 3 & 16462.14 & $\sin \frac{2 \pi x}{a} \sin \frac{3 \pi y}{b}$ \\
\hline 3 & 2 & 16462.14 & $\sin \frac{3 \pi x}{a} \sin \frac{2 \pi y}{b}$ \\
\hline
\end{tabular}

Table 1: Mode shapes and frequency parameters $\lambda_{m n}^{2}$ for

\section{DISCUSSIONS AND CONCLUSIONS}

The eigen value problem of natural (free) vibrations of simply supported Kirchhoff plates have been solved by the Galerkin-Vlasov variational method. The natural frequencies obtained for all the vibrating modes are found to be functions of the material properties and the plate geometry only; and are inherent properties of the elastic Kirchhoff plate; and are independent of any external excitation load. The frequency or characteristic equation was obtained as an infinite number of eigen frequencies constituting the frequency spectrum for the simply supported Kirchhoff plate. The lowest frequency, (fundamental natural frequency) was found for square plates to be

$$
w_{11}=\frac{2 \pi^{2}}{a^{2}} \sqrt{\frac{D}{\rho h}}
$$

The fundamental vibration mode of the simply supported plate is a single sine wave in the $x$ and $y$ coordinate directions. The maximum deflection for the first mode of vibration is found to occur at the plate centre. The fundamental natural frequency is used in the design of plates to avoid resonance failure since resonance occurs when the excitation force vibrates at a frequency that is close to or equal to the natural (eigen) frequency of the plate.

$$
\begin{aligned}
& \text { Let } a / b=k, \text { and let } \\
& \qquad \begin{aligned}
\lambda_{m n}^{2} & =\frac{\rho h a^{4}}{D} \omega_{m n}^{2}=a^{4} \beta_{m n}^{2} \\
\lambda_{m n}^{2} & =\pi^{4}\left(m^{2}+n^{2} k^{2}\right)^{2}
\end{aligned}
\end{aligned}
$$

For a square plate $(\mathrm{k}=1)$

$$
\lambda_{m n}^{2}=a^{4} \beta_{m n}^{2}=\pi^{4}\left(m^{2}+n^{2}\right)^{2}
$$

The non-dimensional frequency parameters and the mode shapes are tabulated in Table 1.

\section{REFERENCES}

[1] Rao, S. Vibration of Continuous Systems, John Wiley and Sons Inc. New Jersey. 2007.

[2] Timoshenko, S. and Woinowsky-Krieger, S. Theory of Plates and Shells. McGraw Hill New York. 1959.

[3] Gorman, D. S. Free vibration analysis of rectangular plates Elsevier North Holland Inc. 1982.

[4] Leissa, A. W. Vibration of Plates Acoustical Society of America. 1993.

[5] Leissa, A. W. The free vibration of rectangular plates Journal of Sound and Vibration, 31, pp 257 - 293. 1973.

[6] Warburton, G. B. The vibrations of rectangular plates. Proceedings of the Institute of Mechanical Engineering Series A. 168 pp. 371 - 384. 1954.

[7] Kirchhoff G. R. Vorlesugen uber mathematisque physic, Mechanic. 1885.

[8] Mindlin R. D. Influence of rotary inertia and shear on flexural vibrations of isotropic, elastic plates. $J$. Appl. Mech. Vol.18 pp 31-38. 1951.

[9] Reissner E. A Twelfth Order Theory of Transverse Bending of Transversely Isotropic Plates $Z A M M, 63$ pp. 285-289. 1983.

[10] Chandrashekhara, K. Theory of plates. University Press (India) Limited. Hyderabad. 2001. 
[11] Szilard, R. Theories and Applications of Plate Analysis. John Wiley and Sons Inc. New Jersey. 2004.

[12] Durban D, Givoli D. and Simmonds J.D. (Editors) Solid Mechanics and its Applications: Advances in the mechanics of plates and shells. The Avionoam Libai Anniversary Klumer Academic Publishers Dordrecht, the Netherlands. 2001.

[13] Ventsel, E. and Krauthammer, T. Thin Plates and Shells: Theory, Analysis and Applications. Marcel Dekker Inc New York, USA. 2001.

[14] Zhou, D. Natural frequencies of rectangular plates using a set of static beam functions in the Rayleigh-
Ritz method. Journal of Sound and Vibration, 189 pp. 81 - 88. 1996.

[15] Ashwin Balasubramanian Plate Analysis with Different Geometrics and Arbitrary Bending Conditions. MSc Thesis in Mechanical Engineering, Faculty of the Graduate School, the University of Texas at Arlington, December, 2011.

[16] Yang Zhoug, Xue-feng Zhao, Hang Liu Vibration of plate on foundation with four edges free by finite cosine integral transform method. Latin American Journal of Solids and Structures, Vol. 11. No.5, October, 2014. 